\title{
COMPUTER IMAGE REGISTRATION TECHNIQUES APPLIED TO NUCLEAR MEDICINE IMAGE
}

\author{
Raquel S. Alves ${ }^{1}$, João Manuel R.S. Tavares ${ }^{2}$ \\ ${ }^{1}$ Faculdade de Engenharia, Universidade do Porto, Portugal \\ ${ }^{2}$ Instituto de Engenharia Mecânica e Gestão Industrial, Departamento de Engenharia \\ Mecânica, Faculdade de Engenharia, Universidade do Porto, Portugal, tavares@fe.up.pt
}

\begin{abstract}
Modern medicine has been using imaging as a fundamental tool in a wide range of applications. Consequently, the interest in automated registration of images from either the same or different modalities has increased. In this chapter, computer techniques of image registration are reviewed, and cover both their classification and the main steps involved. Moreover, the more common geometrical transforms, optimization and interpolation algorithms are described and discussed. The clinical applications examined emphases nuclear medicine.
\end{abstract}

\section{Introduction}

Modern medicine has been using imaging as a fundamental tool to assist in diagnostic procedures, monitoring the evolution of pathologies and planning treatments and surgeries. However, in order to fully exploit digital medical images and their efficient analyses, suitable semi- or full-automated methods of image registration must be developed [1].

Computer techniques of image registration enable the fusion of different medical image modalities and the detection of changes between images acquired from different angles, at different acquisition times or even against an atlas that includes anatomical and functional knowledge. This task of image analysis can also point out changes in size, shape or image intensity over time and compare preoperative images and surgical planned outcomes with the physical world during interventions [2].

The aim of image registration techniques is to find the optimal transformation that best aligns the structures of interest in the input images. Accordingly, the techniques establish the spatial correspondence among features in the images or minimize an error measure or a cost function. To accomplish such goals, optimization algorithms are usually used to find the most suitable geometrical 
transformations, and interpolators are employed to resample the images into the registered discrete spaces.

The more usual applications of image registration techniques in nuclear medicine include correlative image interpretation, attenuation correction, scatter correction, correction for limited resolution and improvement of the reconstruction accuracy in emission tomography. These techniques have also been used in the coregistration of functional studies, for the transformation of images into standard spaces for their comparison against both normal cases and data from other modalities, and in conformal radiotherapy treatment. Also, these methods have been used to improve the interpretation of several functional studies based on static images, including brain, breast, chest, liver, kidneys and colon images, or to assist motion analyses as in cardiac and lung studies.

There have been previous reviews covering medical image registration in general [3-9], medical image classification [10], mutual-information-based registration methods [5], unsupervised registration methods [11], non-rigid image registration $[12,13]$, image registration of nuclear medicine images [14], image registration techniques for specific organs such as breast [15], brain [16, 17] and cardiac images [18]. In this chapter, the classifications of the registration methods suggested by several authors are reviewed. Then, techniques of image registration in general are introduced, including the geometric transforms, similarity measures, optimizers and interpolators. Finally, the main applications related to nuclear medicine imaging are examined.

\section{Registration methods: classification}

There are different classification criteria for image registration techniques depending on the authors. For example, image registration methods were classified into four categories: point methods, edge methods, moment methods and "similarity criterion optimization" methods [19]. Also, a classification based on: data dimensionality, origin of image properties, domain of the transformation, elasticity of the transformations, tightness of property coupling, parameter determination and type of interaction (interactive, semi-automatic or automatic) was proposed [10]. Moreover, registration techniques were also divided into: stereotactic frame systems, point methods, curve and surface methods, moment and principal axes methods, correlation methods, interactive methods, and atlas methods [19].

Registration methods can also be classified according to the subjects and the image modalities involved. Hence, intra-subject and intra-modality applications refer to the image registration of the same subject in images acquired using the same imaging modality. Intra-subject and inter-modality registration is the image registration between images of the same subject but acquired using different imaging modalities, which is a common case that involves Positron Emission Tomography (PET) and Single-Photon Emission Computed Tomography 
(SPECT) images [20]. Inter-subject and intra-modality registration consists of aligning images of different subjects but acquired by the same imaging modality. Finally, inter-subject and inter-modality is related to the alignment of images from different subjects and acquired by different imaging modalities.

Table 1 shows the classification of medical image registration methods that take into account the data dimensionality, nature of the registration basis, the nature and domain of the transformation, type of interaction, optimization procedure, imaging modalities, subject and object involved.

Table 1 Classification of medical image registration methods (adapted from [8]).

\begin{tabular}{|c|c|c|}
\hline $\begin{array}{l}\text { Classification } \\
\text { Criteria }\end{array}$ & & Subdivision \\
\hline \multirow[t]{2}{*}{ Dimensionality } & \multicolumn{2}{|c|}{ Spatial dimension: $2 \mathrm{D} / 2 \mathrm{D}, 2 \mathrm{D} / 3 \mathrm{D}, 3 \mathrm{D} / 3 \mathrm{D}$} \\
\hline & \multicolumn{2}{|l|}{ Temporal series } \\
\hline \multirow{13}{*}{$\begin{array}{l}\text { Nature of the } \\
\text { registration basis }\end{array}$} & \multicolumn{2}{|l|}{ Extrinsic } \\
\hline & \multirow[t]{2}{*}{ Invasive } & Stereotactic frames \\
\hline & & Fiducials (screw markers) \\
\hline & \multirow[t]{2}{*}{ Non-invasive } & Moulds, frames, dental adapters, etc. \\
\hline & & Fiducials (skin markers) \\
\hline & \multicolumn{2}{|l|}{ Intrinsic } \\
\hline & \multirow[t]{2}{*}{$\begin{array}{l}\text { Landmark } \\
\text { based }\end{array}$} & Anatomical \\
\hline & & Geometrical \\
\hline & \multirow[t]{2}{*}{$\begin{array}{l}\text { Segmentation } \\
\text { based }\end{array}$} & Rigid models (points, curves, surfaces, volumes) \\
\hline & & Deformable models (snakes, nets) \\
\hline & \multirow[t]{2}{*}{$\begin{array}{l}\text { Voxel property } \\
\text { based }\end{array}$} & $\begin{array}{l}\text { Reduction to scalar/vectors (moments, principal } \\
\text { axes) }\end{array}$ \\
\hline & & Using full image contents \\
\hline & \multicolumn{2}{|c|}{ Non-image based (calibrated coordinate systems) } \\
\hline
\end{tabular}




\begin{tabular}{|c|c|c|}
\hline Nature of & \multicolumn{2}{|c|}{ Rigid (only rotation and translation) } \\
\hline & \multicolumn{2}{|c|}{ Affine (translation, rotation, scaling and shearing) } \\
\hline & \multicolumn{2}{|l|}{ Projective } \\
\hline & \multicolumn{2}{|l|}{ Curved } \\
\hline \multirow{2}{*}{$\begin{array}{r}\text { Domain of } \\
\text { transformation }\end{array}$} & \multicolumn{2}{|l|}{ Local } \\
\hline & \multicolumn{2}{|l|}{ Global } \\
\hline \multirow[t]{6}{*}{ Interaction } & \multirow[t]{2}{*}{ Interactive } & Initialization supplied \\
\hline & & No initialization supplied \\
\hline & \multirow[t]{3}{*}{$\begin{array}{r}\text { Semi- } \\
\text { automatic }\end{array}$} & User initializing \\
\hline & & User steering/correcting \\
\hline & & Both \\
\hline & \multicolumn{2}{|l|}{ Automatic } \\
\hline \multirow{2}{*}{$\begin{array}{l}\text { Optimization } \\
\text { procedure }\end{array}$} & \multicolumn{2}{|c|}{ Parameters computed directly } \\
\hline & \multicolumn{2}{|c|}{$\begin{array}{l}\text { Parameters searched (the transformation parameters are computed } \\
\text { iteratively using optimization algorithms) }\end{array}$} \\
\hline \multirow{4}{*}{$\begin{array}{c}\text { Imaging } \\
\text { modalities involved }\end{array}$} & \multicolumn{2}{|c|}{ Monomodal } \\
\hline & \multicolumn{2}{|l|}{ Multimodal } \\
\hline & \multicolumn{2}{|c|}{$\begin{array}{l}\text { Modality to model (register the coordinate system of the imaging } \\
\text { equipment with a model coordinate system) }\end{array}$} \\
\hline & \multicolumn{2}{|c|}{$\begin{array}{l}\text { Patient to modality (register the patient with the coordinate system of } \\
\text { the imaging equipment) }\end{array}$} \\
\hline \multirow[t]{3}{*}{ Subject } & \multicolumn{2}{|c|}{ Intra-subject } \\
\hline & \multicolumn{2}{|c|}{ Inter-subject } \\
\hline & \multicolumn{2}{|l|}{ Atlas } \\
\hline
\end{tabular}




\begin{tabular}{|l|l|}
\hline \multirow{2}{*}{ Object } & Head (brain, eye, dental, etc.) \\
\cline { 2 - 3 } & Thorax (entire, cardiac, breast, etc.) \\
\cline { 2 - 3 } & Abdomen (general, kidney, liver, etc.) \\
\cline { 2 - 2 } & Limbs \\
\cline { 2 - 2 } & Pelvis and perineum \\
\hline & Spine and vertebrae \\
\hline
\end{tabular}

Registration methods based on pixel (or voxels in 3D) intensity are known as intensity based, while those based on the geometrical structures extracted from the images as feature or geometrical based; furthermore, frequency or Fourier based registration techniques use the image in the frequency domain and the Fourier transform properties. Feature space information, or techniques based on the amount of image information used, is another classification proposed in the literature [8].

\section{Image registration}

Methods of image registration aim to find the optimal transformation that best aligns the structures of interest in the input images [21-23]. After the attribution of a common coordinate system, the images are transformed into this system. Usually, the registration methods are based on geometric approaches, known as feature-based or intensity-based methods. Feature-based methods start by establishing the correspondence between features in the input images and then compute the geometrical transformation that aligns these features. Intensity-based methods iteratively adjust the transformation that aligns the input images taking into account the intensity of the image pixels (or voxels in 3D), through the minimization of a cost function. Usually, the cost function consists of a similarity measure, i.e. the registration algorithms try to minimize an error measure [24]. In such approaches, optimization algorithms are needed to find the most suitable geometrical transformation, and interpolators are employed to resample the image data into the new common discrete space.

Landmark-based registration methods are based on the identification of the correspondence between landmarks in the two input images. These markers can be distinguished as extrinsic, anatomical and geometrical landmarks. External landmarks are well suited for validation studies; however, their routine application 
can be impracticable, since patient studies may be realized on different days and the location of the markers must not vary during a study. On the other hand, internal anatomical landmarks do not need marker preparation, but in common cases it is difficult to obtain a reliable and accurate localization; hence, they are not used in routine nuclear medicine, but just to access the efficiency of the registration methods. Geometrical landmarks consist of corners and other geometric features that can be identified automatically in the images, however, these features usually present low resolution and low signal-to-noise levels in nuclear medicine images [14]. These problems can be partially overcome using image registration algorithms based on different combinations of landmark-, surface-, attenuation- and intensity-based registration approaches [25, 26].

Boundaries or surfaces are more distinct in medical images than the usual simpler landmarks, and are therefore a valuable tool for registration methods based on surfaces. These methods require the establishment of correspondence between boundaries or surfaces that are defined in the input images, and they give good results in inter-modality registration, where both images can have very different pixel (or voxel) values [14]. There are four methods to carry out a surface registration, namely: feature, point, model and global similarity based methods. The criterion for selecting one of these is application-specific. The size of the transformation to be computed and its nature are also factors of choice [27].

Feature-based methods enable building explicit models of distinguishable anatomical elements in each image such as surfaces, curves and point landmarks, which can be aligned with their counterparts in the second image. The use of feature-based methods is recommended for images that contain enough distinctive and easily detectable features [15]. Figure 1 illustrates a typical feature-based registration algorithm.

Hybrid registration, using combined surface and volumetric-based registration methods, enables the extraction of relevant geometrical information from surfacebased morphing and its following diffusion into the volume [28]. Surface alignment has been employed, for example, in image-guided surgery [29].

On the other hand, intensity based registration techniques align intensity patterns using mathematical or statistical criteria over the whole image without considering anatomical information. Combining geometric features and intensity features in registration should result in a more robust method. Therefore, hybrid algorithms involving intensity-based and model-based criteria allow the establishment of more accurate alignments, since these methods tend to average the error caused by noise or random fluctuations [12]. Figure 2 presents the general framework of the registration methods based on the minimization of a cost function. Image registration algorithms can also perform image correction by using the intensities of pixels (or voxels), locally or globally, in the two input images [30]. 


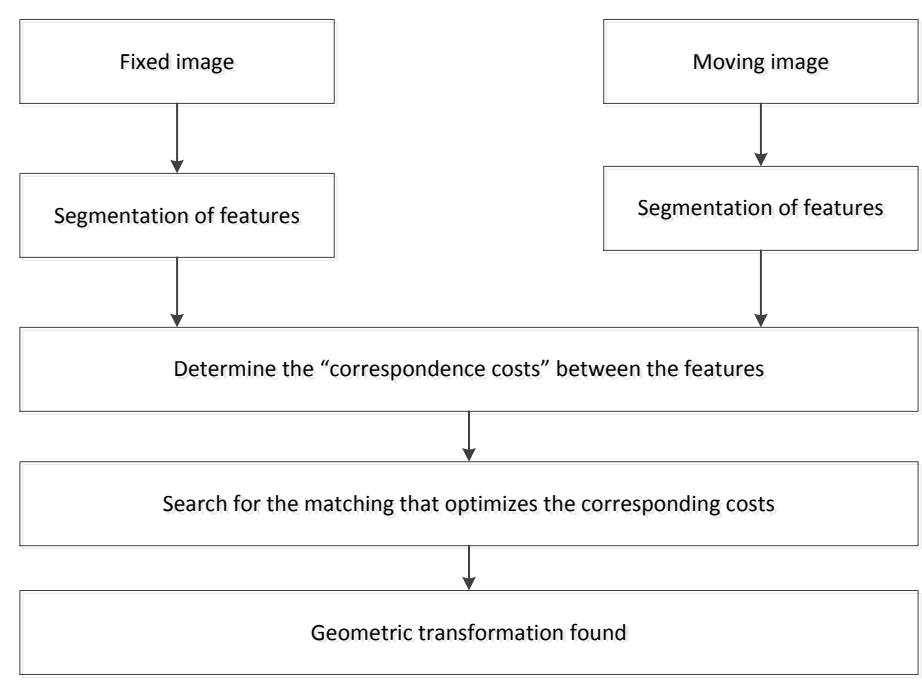

Figure 1 Diagram of a typical feature-based registration algorithm

Image pre-processing is generally used before the registration to ensure that a suitable registration solution is successfully achieved, since it provides an enhanced definition of the object boundaries, and it enables intensity remapping in order to modify the range of the intensities that are used by the registration algorithms. However, it is fundamental that the pre-processing algorithms do not change the original images excessively and are not too time-consuming [14].

Rigid and affine registrations can be found in seconds; contrarily, non-rigid registrations can take minutes or hours [12]. Therefore, it is important to improve the speed of image registration techniques. Coarse-to-fine methods are commonly used, as they initially provide fast estimates and then gradually better-quality ones. Another solution to reduce the required registration time consists in sub-sampling the original images, involving spatial domain- or intensity-based procedures, and increase the image resolutions as the registration algorithm gets closer to the final solution [14]. 


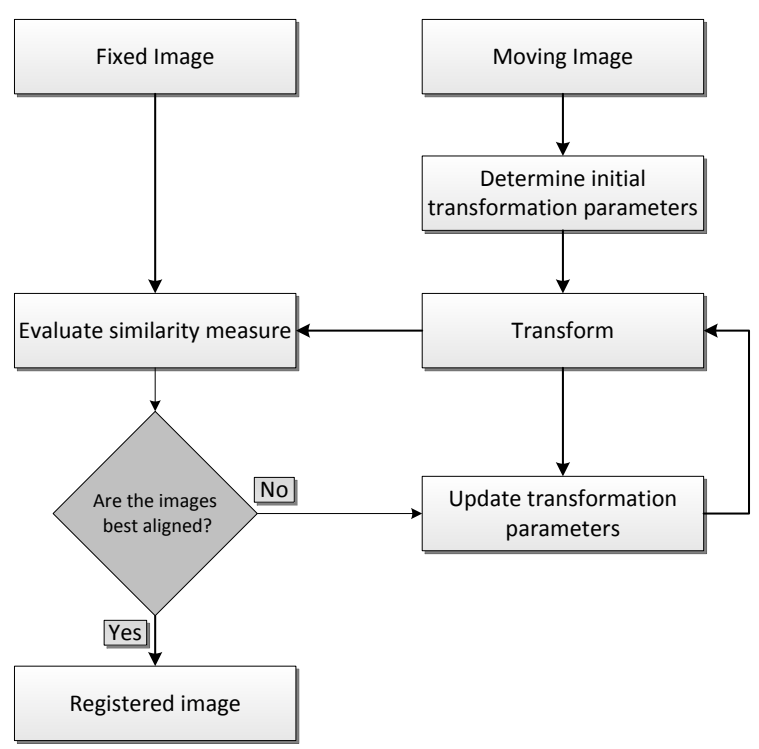

Figure 2 General scheme of the image registration methods based on the optimization of a cost function (adapted from [14])

\subsection{Geometric transformations}

The goal of image registration algorithms is to find the transform involved between the two input images by means of geometrical transformations, whose number of parameters varies with the complexity of the transformation model used. The selection of the appropriate geometrical transformation model is crucial to the success of the registration process.

The geometrical transformation model can lead to rigid or non-rigid registrations. The simplest geometrical transformation model is based on a rigid transform that only considers rotations and translations, which is applied to all elements of one of the input images, usually known as moving images. Affine transform models include translations, rotations, scaling and shearing so that the straight lines of one image are kept as straight lines in a second image, and the parallel lines are preserved parallel [4]. An identity transformation maintains all the elements of the input image in their original configuration. Fig. 3 illustrates these three types of transformation using squares. It should be noted that, a more complex transformation model implies a higher number of degrees of freedom leading to non-rigid transformations. 

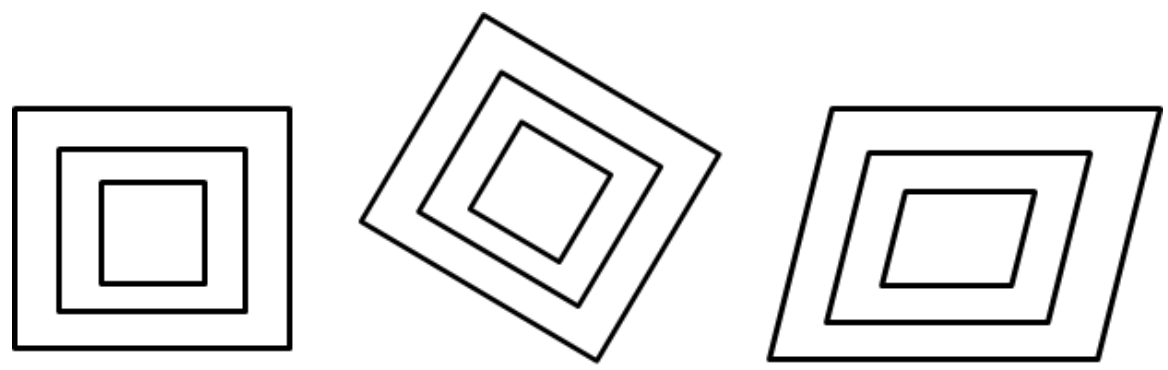

Figure 3 Three types of geometrical transformations applied to squares: identity transformation (left), rigid transformation (middle) and affine transformation (right).

Image registration algorithms based on non-rigid transformations are required, for example, when the alignment between images of one individual and an atlas needs to be established [31], or when substantial anatomical variability among individuals needs to be accommodated [12, 13, 32, 33]. When compared with rigid transformations, non-rigid based registration algorithms have a higher number of degrees of freedom $[34,35]$. They are frequently used in image registration when the image acquisition parameters are not known [36], and usually include an initial rigid body or affine transformation that provides an initial solution for the transformation. Hence, a good pre-registration method is recommended to obtain an initial position and orientation closer to the optima non-rigid registration solution. However, a higher number of parameters in the transformation model can introduce undesirable transformations and therefore, a regularization term must be taken into account [37-39]. Non-rigid image transformations can be achieved using basis functions such as a set of Fourier [40-43] or Wavelet basis functions [44].

Image registration using splines can be achieved with techniques based on the assumption that a set of control points are mapped into the target image from their corresponding counterparts in the source image [45], and a displacement field can be established and interpolated [46]. Therefore, spline-based geometrical transformations either interpolate or approximate the displacements at control points. Thin-plate splines (TPS) are based on radial basis functions and are used in surface interpolation of scattered data [32,33]. Each basis function contributes to the transformation, and each control point has a global influence on the transformation. The modelling of local deformations can be more difficult with these functions, which requires the use of free-form transformations based on locally controlled functions [47, 48]. B-splines deform an object through the manipulation of an underlying mesh of control points generating a smooth continuous transformation. Thin-Plate Spline Robust Point Matching (TPS-RPM) algorithms have been used for non-rigid registration, showing robustness when aligning models with a large number of outliers [46].

Elastic, deformable or curved registration methods enable deforming and resampling similar to the stretching of an elastic material. Their limitations are because of the highly localized deformations that cannot be modelled due to stress 
deformation energy [45]. In the literature, there are reviews about the most promising non-linear registration strategies currently used in medical imaging, such as a novel curvature based registration technique that permits a faster image registration [51], the application of a deformable registration method in the automated hexahedral meshing of anatomical structures [52, 53], symmetric nonrigid registration [54] and Brownian Warps, which is a diffeomorphism registration algorithm [55]. Also fluid registration and registration using optical flow are approaches that are equivalent to the equation of motion for incompressible flow [45].

\subsection{Similarity measures}

The characteristics of the image modalities and the level of misregistration must be taken into account on choosing the similarity measure. Similarity measures can be classified into feature or intensity based metrics; however, some similarity metrics can be included in both classes. The similarity measure used in deformable image registration is commonly constituted by one term related to the pixel (or voxel in 3-D) intensity or to the matching between the structures in the images, and another one related to the deformation. Then, the cost function built is a trade-off between the pixel (or voxel) intensity or matching between the structures and the constraints imposed on the deformation field.

Concerning the feature based measures; the similarity measure commonly used represents the average distance between the corresponding features. Similarly, surfaces or edges based measures quantify an average distance between the corresponding surfaces, or between a surface extracted in one image and its corresponding set of points in the other image [9].

The simplest similarity measure compares the intensity values between the input images directly [14]. To register intra-subject and intra-modality images, the Correlation Coefficient (CC) has been an adequate similarity measure, since it involves the multiplication of the corresponding image intensities assuming a linear relationship between the intensity values. However, it is possible to subtract the corresponding image intensities instead of multiplying them, thus the search for the best alignment is based on the Smallest Sum of Squared Intensity Differences (SSD). However, due to the sensitiveness of SSD to a small number of voxels that have very large intensity differences between the input images [45], the Sum of Absolute Differences (SAD) is usually employed instead, as shown in Figure 4 [56].

\begin{tabular}{|c|c|c|}
\hline Image \#1 & Image \#2 & $\begin{array}{c}\text { SAD values between } \\
\text { images \#1 and \#2 }\end{array}$ \\
\hline
\end{tabular}



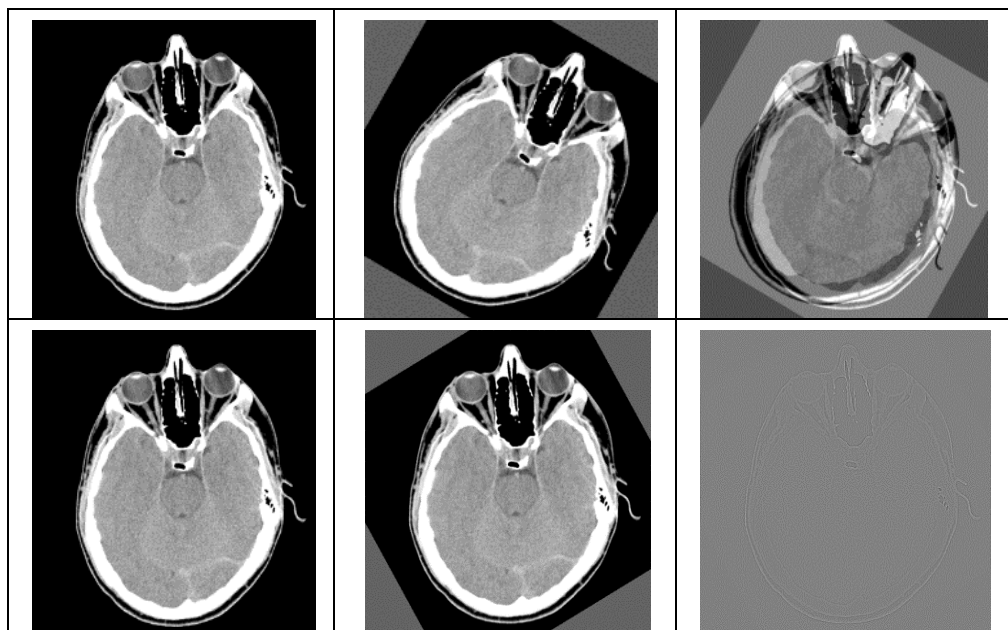

Figure 4 Application of SAD to highlight the differences between the two images, before registration (top) and after registration (bottom). Before the registration, the original images present large absolute differences (top-right image), while after the registration, the aligned images present low or zero absolute differences (bottom-right image). Thus, the similarity measure indicates how well the images are aligned.

Ratio Image Uniformity (RIU), also known as Variance of Intensity Ratios (VIR), is an iterative technique similar to derived ratio images. The uniformity of the ratio image is quantified as the normalized standard deviation of the respective pixels [45]. This technique is used to find the transform that maximizes that uniformity. These similarity measures are used for intra-modality registration. Partitioned Intensity Uniformity (PIU) seeks to maximize the uniformity by minimizing the normalized standard deviation, and is usually used to register inter-modality images [4].

Image registration algorithms have also been developed based on information theory to solve both inter- and intra-modality registration problems. This image registration approach can be described as trying to maximize the amount of shared information between the two input images, which means that information can be used as a registration metric [57]. The joint entropy measures the amount of information existing in the combined images, and it has been used for rigid and non-rigid image registration [48, 49]. Mutual information can be given by the difference between the sum of entropies of the individual images at the overlap regions and the joint entropy of the combined images [58]. Hence, it is the measure of how one image explains the other $[45,58,60]$ and makes no assumptions about the functional form or relationship between the image intensities. Changes in very low intensity overlapped regions, such as those due to noise, can disproportionally contribute to artefacts [45] that affect the registration accuracy when based on mutual information, so this method is commonly used combined with the normalization of the joint entropy $[45,59]$. 


\subsection{Optimization}

All the registration algorithms based on optimization require an iterative approach, whose initial estimate of the transformation is gradually refined by trial and error, by calculating the similarity measure, or cost function, at each iteration. So, the optimization process consists of both estimating the transformation and evaluating the similarity measure till the algorithm converges to a point when no new transformation can be found with a better similarity measure value [4]. Hence, the optimization algorithm evaluates the value of the similarity measure, searching for the subsequent alignment transformations that will end the registration process if an optimal value is reached. In other words, the registration is achieved by searching the transformation that increases or decreases the cost function until a maximum or minimum is found, depending on the type of the cost function used. The optimization process is based on the fact that the quality of the matching of two images is balanced against some constraint. This constraint has the purpose of prohibiting implausible deformations and may be provided, for example, by some estimate of the energy required to physically induce the deformation [45].

One of the major difficulties of the registration methods is that the optimization algorithms can converge to an incorrect local optimum, because multiple optima can exist within the space of the transformation parameters [60-62]. The erroneous optima can be due to interpolation artefacts or good local matches between features or intensities; however it can be avoided by smoothing the original images. Also, the position and orientation associated to the two input images must be sufficiently close so the algorithm converges to the best solution within its functional range [45]. To choose the solution that has the best function cost value, a multi-start optimization can be used to get the global optimal solution $[45,60]$. Additionally, the images can be initially registered at low resolution and then the transformation obtained is used as the starting transformation for registration at a higher resolution $[63,64]$.

\subsection{Interpolation}

A process of interpolation is commonly applied to transform an image space into the space of another image in order to register them; i.e. when it is necessary to estimate the values of the transformed image [14]. Thus, its goal is to estimate the intensity at the new position [8] and depends on the motivation for registering the images. The accuracy and speed of the registration process can be improved through the use of suitable interpolation solutions.

Nearest neighbour, linear interpolation or trilinear interpolation are the simplest interpolation methods, and consist of curve fitting, using linear polynomials. The interpolated image will be smoother than the original. When the interpolation complexity increases, the number of polynomial variables also increases and the 
smoothing effect can be more severe or even generate artefacts [14]. Recent interpolation methods between neighbouring image slices in grey-scale are based on B-splines [65], geometric multi-grid [66], using a modified control grid interpolation algorithm [67] or adaptive 2D autoregressive modelling and softdecision estimation [68].

The interpolation error can introduce modulations in the similarity measure used in the registration process, since the transformations involve pure translations of images with equal sampling spacing, and the period of the modulation is the same as the sampling spacing. Interpolation methods must be used with a practicable computational cost; for example, by using a low cost interpolation as trilinear or nearest neighbour first. Hence, it is a good practice to employ a more expensive interpolation approach just in the last iterations of the registration process or even take advantage of the spatial-frequency dependence of the interpolation error, by using, for example, cubic B-splines or windowed sinc interpolators. Finally, the use of a more robust interpolation solution in the optimization step may be imposed if the level of smoothness and robustness of the similarity measure is affected by interpolation imperfections [69].

\section{Accuracy Assessment and Validation}

The image registration methods must be validated, especially in medical applications. A verification process based on the comparison of the results obtained against a gold standard must be applied. Additionally, any process of accuracy assessment and validation should have a very low failure rate and be very accurate.

The visual assessment of registered images has been used as a standard method; however, this depends heavily on the clinical experience of the observers, besides being subject to inter- and intra-observer variability. To overcome this disadvantage, the software industry has already developed standards, protocols and quality assessment procedures [70]. The validation usually follows a sequence of measurements using computer-generated models, known as software generated phantoms [71], and the comparison of patients' images against the registration algorithms must be efficient. In order to extend the experimental validation of an image registration system to a clinical situation [72], the target registration error (TRE), which is a measure of error, is recommended to be used to monitor the clinical validation process [55], since it evaluates a target feature [73]. However, this can vary depending on the application, since there are different image modalities, anatomical structures and pathologies, and distinct positions within a view [74]. Several fiducial features can be used as registration cues, indicating the registration accuracy; this is a desirable method for rigid-body registration. Validation is accomplished by establishing statistical relationships between fiducial localization error (FLE) and TRE [75] to translate self-consistency into accuracy [74]. Furthermore, based on registration circuits, another self- 
consistency method [74] has also been considered, where a set of three or more images are registered in pairs.

The efforts to improve the registration validation methods have been focussed more on rigid registration than on non-rigid registration [74]. Improvement in these methods is fundamental for novel registration models to be accepted as a clinical tool, which is impossible without an optimal validation method.

\section{Registration in Nuclear Medical Imaging}

Nuclear image modalities have been widely used in healthcare diagnostics. They provide physiological diagnoses through the use of radiotracers to map the metabolism and fluid flow in tissues, organs or organ systems [76]. Nuclear medicine benefits from such integration and image registration plays a central role in this integration [18,77-79].

In oncology, the completion of the medical Positron Emission Tomography (PET) examination, usually hybrid Positron Emission Tomography/Computed Tomography (PET/CT) $[80,81]$ enables the detection of tumours at early stages, As this exam is capable of detecting the development of a cancer it can help in the proper choice for the treatment and the later evaluation of the therapeutic response.

In cardiology, several studies have been developed, particularly in the study of chronic ischemia [20, 82-85], myocardial perfusion [18-20, 86-96], atherosclerosis rate [85,97], post-transplantation [18] and cardiac nervous system. Registration of cardiac images is more complex than the registration of images of static organs, since it is a non-rigid moving organ inside a non-static body, and exhibits few easily distinguishable and accurate landmarks [18]. Non-rigid registration is, for example, a key requirement for the application of cardiac function biomechanical models, through the building of a generic cardiac model that is instantiated by linear elastic registration with cardiac images of a subject acquired using different modalities [12].

As regards the neurological and psychiatric disorders, molecular imaging registration has the ability to reveal non-detectable lesions by other imaging methods [98], and provides information on the physiological and biochemical properties and subsequent functional integrity of brain damaged adjacent regions [99]. The pre-surgical evaluation of epilepsy [14, 57] and guided biopsy in brain tumours [19], evaluation of primary brain tumours, dementia diagnosis and selection of stroke patients for surgical treatment [99] are usually based on the quantification of regions of interest in nuclear medicine images. Such quantification can be automated using techniques of image processing and analysis; such as image registration techniques for image correction. These techniques also allow the study of Parkinson's disease [85, 99], Alzheimer [100, 101], and movement disorders [102]. Monitoring changes in the individual by acquiring series of imaging scans and highlighting differences using image 
registration is a common practice and it is particularly useful in dementia where fluid registration is a cue to visualize patterns of regional atrophies [12].

Fully automatic multimodality image registration algorithms are also employed for aligning functional data with anatomic information, such as Magnetic Resonance/PET (MR/PET), Computerized Tomography/PET (CT/PET), and MR/SPECT inter-modality registrations.

\section{Conclusions}

Most current algorithms for medical image registration use rigid body transformations or affine transformations, and are restricted to parts of the body where the tissue deformations are small compared with the desired registration accuracy. Algorithms based on optimizing of a similarity measure and based on information theory can be applied automatically to a variety of imaging modality combinations, without the need of pre-segmenting the images, and can be extended to non-affine transformations. However, it is recommended to preregister the input images with an image registration technique based on rigid transformation, and then finalize the process using another image registration technique based on deformable transformations.

Fully-automated inter-modality registration is still unusual in normal clinical practice, but this kind of image registration is being used in medical research, especially in neurosciences, where it is used in functional studies, in cohort studies and to quantify changes in structures during ageing and the development of diseases. However, its clinical use has logistical difficulties due to the need to acquire and register a large number of images in a reduced period, requiring advanced computational infrastructures as well as the storing of vast amounts of image data.

Due to the functional diagnosis that molecular imaging provides, computer techniques to register SPECT and PET images have been applied in clinical diagnosis, in order to assess the response to treatments and the delivery of targeted therapies. Image registration has proved its potential to aid the medical diagnosis, surgery and therapy. Examples include the combination of functional and high anatomical information to assist the localization and determination of abnormalities and the planning of their treatment. Besides, differences between two medical exams can be directly quantified, providing more objective evidences of the effects of intervention or responses to therapy in successive studies. 


\section{Acknowledgment}

This work was partially done in the scope of the project with reference PTDC/BBB-BMD/3088/2012, financially supported by Fundação para a Ciência e a Tecnologia (FCT), in Portugal. 


\section{References}

1. Rao, A., Chandrashekara, R., Sanchez-Ortiz, G.I., Mohiaddin, R., Aljabar, P., Hajnal, J. V., Puri, B.K. \& Rueckert, D. (2004). Spatial transformation of motion and deformation fields using nonrigid registration. Medical Imaging, IEEE Transactions on, 23(9), 1065-1076.

2. Fox, J.L., Rengan, R., O’Meara, W., Yorke, E., Erdi, Y., Nehmeh, S., Leibel, S. A. \& Rosenzweig, K.E. (2005). Does registration of PET and planning CT images decrease interobserver and intraobserver variation in delineating tumor volumes for non-small-cell lung cancer?. International Journal of Radiation Oncology* Biology* Physics, 62(1), 70-75.

3. Maintz JBA, Viergever MA (1998) A survey of medical image registration methods. 2:1-36.

4. Hill, D. L., Batchelor, P. G., Holden, M., \& Hawkes, D. J. (2001). Medical image registration. Physics in medicine and biology, 46(3), R1.

5. Pluim, J. P., \& Fitzpatrick, J. M. (2003). Image registration. Medical Imaging, IEEE Transactions on, 22(11), 1341-1343.

6. Zitova, B., \& Flusser, J. (2003). Image registration methods: a survey. Image and vision computing, 21(11), 977-1000.

7. Oliveira, F. P., Sousa, A., Santos, R., \& Tavares, J. M. R. (2012). Towards an efficient and robust foot classification from pedobarographic images. Computer methods in biomechanics and biomedical engineering, 15(11), 1181-1188.

8. Oliveira, F. P., \& Tavares, J. M. R. (2014). Medical image registration: a review. Computer methods in biomechanics and biomedical engineering, 17(2), 73-93.

9. Hutton, B. F., \& Braun, M. (2003, July). Software for image registration: algorithms, accuracy, efficacy. In Seminars in nuclear medicine (Vol. 33, No. 3, pp. 180-192). WB Saunders.

10. Van den Elsen, P. A., Pol, E. J., \& Viergever, M. A. (1993). Medical image matching-a review with classification. Engineering in Medicine and Biology Magazine, IEEE, 12(1), 26-39.

11. Zhang, H., Fritts, J. E., \& Goldman, S. A. (2008). Image segmentation evaluation: A survey of unsupervised methods. computer vision and image understanding, 110(2), 260-280.

12. Crum WR (2004) Non-rigid image registration: theory and practice. British Journal of Radiology 77, S140-S153.

13. Holden, M. (2008). A review of geometric transformations for nonrigid body registration. Medical Imaging, IEEE Transactions on, 27(1), 111-128.

14. Hutton, B. F., Braun, M., Thurfjell, L., \& Lau, D. Y. (2002). Image registration: an essential tool for nuclear medicine. European journal of nuclear medicine and molecular imaging, 29(4), 559-577.

15. Guo, Y., Sivaramakrishna, R., Lu, C. C., Suri, J. S., \& Laxminarayan, S. (2006). Breast image registration techniques: a survey. Medical and Biological Engineering and Computing, 44(1-2), 15-26.

16. Toga, A. W., \& Thompson, P. M. (2001). The role of image registration in brain mapping. Image and vision computing, 19(1), 3-24. 
17. Gholipour, A., Kehtarnavaz, N., Briggs, R., Devous, M., \& Gopinath, K. (2007). Brain functional localization: a survey of image registration techniques. Medical Imaging, IEEE Transactions on, 26(4), 427-451.

18. Mariani, G., Bruselli, L., Kuwert, T., Kim, E.E., Flotats, A., Israel, O., Dondi, M. \& Watanabe, N.(2010). A review on the clinical uses of SPECT/CT. European journal of nuclear medicine and molecular imaging, 37(10), 1959-1985.

19. Maurer, C. R., \& Fitzpatrick, J. M. (1993). A review of medical image registration. Interactive image-guided neurosurgery, 17.

20. Declerck, J., Feldmar, J., Goris, M. L., \& Betting, F. (1997). Automatic registration and alignment on a template of cardiac stress and rest reoriented SPECT images. Medical Imaging, IEEE Transactions on, 16(6), 727-737.

21. Lee, E., \& Gunzburger, M. (2010). An optimal control formulation of an image registration problem. Journal of mathematical imaging and vision, 36(1), 69-80.

22. Shafique, K., \& Shah, M. (2005). A noniterative greedy algorithm for multiframe point correspondence. Pattern Analysis and Machine Intelligence, IEEE Transactions on, 27(1), 51-65.

23. Shapiro, L. S., \& Michael Brady, J. (1992). Feature-based correspondence: an eigenvector approach. Image and vision computing, 10(5), 283-288.

24. Leclerc, Y. G., Luong, Q. T., \& Fua, P. (2003). Self-consistency and MDL: A paradigm for evaluating point-correspondence algorithms, and its application to detecting changes in surface elevation. International Journal of Computer Vision, 51(1), 63-83.

25. Johnson, H. J., \& Christensen, G. E. (2002). Consistent landmark and intensity-based image registration. Medical Imaging, IEEE Transactions on, 21(5), 450-461.

26. Betke, M., Hong, H., Thomas, D., Prince, C., \& Ko, J. P. (2003). Landmark detection in the chest and registration of lung surfaces with an application to nodule registration. Medical Image Analysis, $7(3), 265-281$.

27. Audette, M. A., Ferrie, F. P., \& Peters, T. M. (2000). An algorithmic overview of surface registration techniques for medical imaging. Medical Image Analysis, 4(3), 201-217.

28. Postelnicu, G., Zollei, L., \& Fischl, B. (2009). Combined volumetric and surface registration. Medical Imaging, IEEE Transactions on, 28(4), 508-522.

29. Herline, A. J., Herring, J. L., Stefansic, J. D., Chapman, W. C., Galloway, R. L., \& Dawant, B. M. (2000). Surface registration for use in interactive, image-guided liver surgery. Computer Aided Surgery, 5(1), 11-17.

30. Jia, J., \& Tang, C. K. (2005). Tensor voting for image correction by global and local intensity alignment. Pattern Analysis and Machine Intelligence, IEEE Transactions on, 27(1), 36-50.

31. Hurvitz, A., \& Joskowicz, L. (2008). Registration of a CT-like atlas to fluoroscopic X-ray images using intensity correspondences. International journal of computer assisted radiology and surgery, 3(6), 493-504. 
32. Wu, C., Murtha, P. E., \& Jaramaz, B. (2009). Femur statistical atlas construction based on twolevel 3D non-rigid registration. Computer Aided Surgery, 14(4-6), 83-99.

33. Zagorchev, L., \& Goshtasby, A. (2006). A comparative study of transformation functions for nonrigid image registration. Image Processing, IEEE Transactions on, 15(3), 529-538.

34. Bronstein, A. M., Bronstein, M. M., \& Kimmel, R. (2009). Topology-invariant similarity of nonrigid shapes. International journal of computer vision, 81(3), 281-301.

35. Budd, C., Huang, P., Klaudiny, M., \& Hilton, A. (2013). Global non-rigid alignment of surface sequences. International Journal of Computer Vision, 102(1-3), 256-270.

36. Kadyrov, A., \& Petrou, M. (2006). Affine parameter estimation from the trace transform. Pattern Analysis and Machine Intelligence, IEEE Transactions on, 28(10), 1631-1645.

37. Ramsay, J. O., Hooker, G., Campbell, D., \& Cao, J. (2007). Parameter estimation for differential equations: a generalized smoothing approach. Journal of the Royal Statistical Society: Series B (Statistical Methodology), 69(5), 741-796.

38. Stefanescu, R., Pennec, X., \& Ayache, N. (2004). Grid powered nonlinear image registration with locally adaptive regularization. Medical image analysis, 8(3), 325-342.

39. Sorzano, C. O., Thevenaz, P., \& Unser, M. (2005). Elastic registration of biological images using vector-spline regularization. Biomedical Engineering, IEEE Transactions on, 52(4), 652-663.

40. Andreetto, M., Cortelazzo, G. M., \& Lucchese, L. (2004, September). Frequency domain registration of computer tomography data. In 3D Data Processing, Visualization and Transmission, 2004. 3DPVT 2004. Proceedings. 2nd International Symposium on (pp. 550-557). IEEE.

41. Larrey-Ruiz, J., Verdú-Monedero, R., \& Morales-Sánchez, J. (2008). A fourier domain framework for variational image registration. Journal of Mathematical Imaging and Vision, 32(1), 57-72.

42. Oliveira, F. P., Pataky, T. C., \& Tavares, J. M. R. (2010). Registration of pedobarographic image data in the frequency domain. Computer methods in biomechanics and biomedical engineering, 13(6), $731-740$.

43. Pan, W., Qin, K., \& Chen, Y. (2009). An adaptable-multilayer fractional Fourier transform approach for image registration. Pattern Analysis and Machine Intelligence, IEEE Transactions on, $31(3), 400-414$.

44. Gefen, S., Tretiak, O., Bertrand, L., Rosen, G. D., \& Nissanov, J. (2004). Surface alignment of an elastic body using a multiresolution wavelet representation. Biomedical Engineering, IEEE Transactions on, 51(7), 1230-1241.

45. Hajnal, J., Hill, D. L. G., \& Hawkes, D. J. Medical image registration. (2001). Non rigid registration: concepts, algorithms and applications, 281-302.

46. Rueckert, D. (2001). Nonrigid registration: Concepts, algorithms, and applications. Medical Image Registration, 281-301.

47. Rogers, M., \& Graham, J. (2007). Robust and accurate registration of 2-D electrophoresis gels using point-matching. Image Processing, IEEE Transactions on, 16(3), 624-635. 
48. Šerifovic-Trbalic, A., Demirovic, D., Prljaca, N., Székely, G., \& Cattin, P. C. (2009). Intensitybased elastic registration incorporating anisotropic landmark errors and rotational information. International journal of computer assisted radiology and surgery, 4(5), 463-468.

49. Bayro-Corrochano, E., \& Rivera-Rovelo, J. (2009). The use of geometric algebra for 3D modeling and registration of medical data. Journal of Mathematical Imaging and Vision, 34(1), 48-60.

50. Reyes-Lozano, L., Medioni, G., \& Bayro-Carrochano, E. (2010). Registration of 2d points using geometric algebra and tensor voting. Journal of Mathematical Imaging and Vision, 37(3), 249-266.

51. Fischer, B., \& Modersitzki, J. (2004). A unified approach to fast image registration and a new curvature based registration technique. Linear Algebra and its applications, 380, 107-124.

52. Kybic, J., \& Unser, M. (2003). Fast parametric elastic image registration. Image Processing, IEEE Transactions on, 12(11), 1427-1442.

53. Grosland, N. M., Bafna, R., \& Magnotta, V. A. (2009). Automated hexahedral meshing of anatomic structures using deformable registration. Computer methods in biomechanics and biomedical engineering, 12(1), 35-43.

54. Tagare, H. D., Groisser, D., \& Skrinjar, O. (2009). Symmetric non-rigid registration: A geometric theory and some numerical techniques. Journal of Mathematical Imaging and Vision, 34(1), 61-88.

55. Nielsen, M., Johansen, P., Jackson, A. D., Lautrup, B., \& Hauberg, S. (2008). Brownian warps for non-rigid registration. Journal of Mathematical Imaging and Vision, 31(2-3), 221-231.

56. Sonka, M., \& Fitzpatrick, J. M. (2000). Handbook of medical imaging (Volume 2, Medical image processing and analysis). SPIE- The international society for optical engineering.

57. Hoffer, P. B. (1995). Difference Images Calculated from Ictal and Interictal Technetium-. 99mHMPAO SPECIT Scans of Epilepsy.

58. Pluim, J. P., Maintz, J. A., \& Viergever, M. A. (2003). Mutual-information-based registration of medical images: a survey. Medical Imaging, IEEE Transactions on, 22(8), 986-1004.

59. Seppa, M. (2008). Continuous sampling in mutual-information registration. Image Processing, IEEE Transactions on, 17(5), 823-826.

60. Collignon, A., Maes, F., Delaere, D., Vandermeulen, D., Suetens, P., \& Marchal, G. (1995, June). Automated multi-modality image registration based on information theory. In Information processing in medical imaging (Vol. 3, No. 6, pp. 263-274).

61. Marques, J. S., \& Abrantes, A. J. (1997). Shape alignment-optimal initial point and pose estimation. Pattern Recognition Letters, 18(1), 49-53.

62. Nguyen, M. H., \& De la Torre, F. (2010). Metric learning for image alignment. International Journal of Computer Vision, 88(1), 69-84.

63. Marai, G. E., Laidlaw, D. H., \& Crisco, J. J. (2006). Super-resolution registration using tissueclassified distance fields. Medical Imaging, IEEE Transactions on, 25(2), 177-187. 
64. Telenczuk, B., Ledesma-Carbato, M. J., Velazquez-Muriel, J. A., Sorzano, C. O. S., Carazo, J. M., \& Santos, A. (2006, April). Molecular image registration using mutual information and differential evolution optimization. In Biomedical Imaging: Nano to Macro, 2006. 3rd IEEE International Symposium on (pp. 844-847). IEEE.

65. Penney, G. P., Schnabel, J. A., Rueckert, D., Viergever, M. A., \& Niessen, W. J. (2004). Registration-based interpolation. Medical Imaging, IEEE Transactions on, 23(7), 922-926.

66. Keeling, S. L. (2007). Generalized rigid and generalized affine image registration and interpolation by geometric multigrid. Journal of Mathematical Imaging and Vision, 29(2-3), 163-183.

67. Frakes, D. H., Dasi, L. P., Pekkan, K., Kitajima, H. D., Sundareswaran, K., Yoganathan, A. P., \& Smith, M. J. (2008). A new method for registration-based medical image interpolation. Medical Imaging, IEEE Transactions on, 27(3), 370-377.

68. Zhang, X., \& Wu, X. (2008). Image interpolation by adaptive 2-D autoregressive modeling and soft-decision estimation. Image Processing, IEEE Transactions on, 17(6), 887-896.

69. Oliveira, F. P., \& Tavares, J. M. R. (2014). Medical image registration: a review. Computer methods in biomechanics and biomedical engineering, 17(2), 73-93.

70. Lee, J. S., Park, K. S., Lee, D. S., Lee, C. W., Chung, J. K., \& Lee, M. C. (2005). Development and applications of a software for Functional Image Registration (FIRE). Computer methods and programs in biomedicine, 78(2), 157-164.

71. Dickson, J. C., Tossici-Bolt, L., Sera, T., Erlandsson, K., Varrone, A., Tatsch, K., \& Hutton, B. F. (2010). The impact of reconstruction method on the quantification of DaTSCAN images. European journal of nuclear medicine and molecular imaging, 37(1), 23-35.

72. Mutic, S., Dempsey, J.F., Bosch, W.R., Low, D.A., Drzymala, R.E., Chao, K.S., Goddu, S.M., Cutler, P.D. \& Purdy, J.A. (2001). Multimodality image registration quality assurance for conformal three-dimensional treatment planning. International Journal of Radiation Oncology* Biology* Physics, 51(1), 255-260.

73.Wiles AD, Likholyot A, Frantz DD, Peters TM (2008). A statistical model for point-based target registration error with anisotropic fiducial localizer error. IEEE transactions on medical imaging 27:378-90.

74. Oliveira, F. P., \& Tavares, J. M. R. (2011). Novel framework for registration of pedobarographic image data. Medical \& biological engineering \& computing,49(3), 313-323.

75. Nicolau, S., Pennec, X., Soler, L., \& Ayache, N. (2003). Evaluation of a new 3D/2D registration criterion for liver radio-frequencies guided by augmented reality. In Surgery Simulation and Soft Tissue Modeling (pp. 270-283). Springer Berlin Heidelberg.

76. Wahl, R. L. (1999). To AC or not to AC: that is the question. Journal of Nuclear Medicine, 40(12), 2025-2028.

77. Stokking, R., Zuiderveld, K. J., Hulshoff Pol, H. E., Van Rijk, P. P., \& Viergever, M. A. (1997). Normal fusion for three-dimensional integrated visualization of SPECT and magnetic resonance brain images. Journal of Nuclear Medicine, 38(4), 624-629. 
78. Jacene, H. A., Goetze, S., Patel, H., Wahl, R. L., \& Ziessman H. A. (2008). Advantages of Hybrid SPECT/CT vs SPECT alone. Open Med Imag J, 13(2), 67-79.

79. Bhargava, P., (2011). Overview of SPECT / CT Applications. 56th Annual Meeting of Southwestern chapter of the Society of Nuclear Medicine.

80. Townsend, D. W. (2008, May). Positron emission tomography/computed tomography. In Seminars in nuclear medicine (Vol. 38, No. 3, pp. 152-166). WB Saunders.

81. Gerasimou, G. P. (2006). Molecular imaging (SPECT and PET) in the evaluation of patients with movement disorders. Nuclear Medicine Review, 9(2), 147-153.

82. Slart, R. H., Tio, R. A., Zijlstra, F., \& Dierckx, R. A. (2009). Diagnostic pathway of integrated $\mathrm{SPECT} / \mathrm{CT}$ for coronary artery disease. European journal of nuclear medicine and molecular imaging, 36(11), 1829-1834.

83. Xue, Z., Shen, D., \& Davatzikos, C. (2004). Determining correspondence in 3-D MR brain images using attribute vectors as morphological signatures of voxels. Medical Imaging, IEEE Transactions on, 23(10), 1276-1291.

84. Shekhar, R., Zagrodsky, V., Castro-Pareja, C. R., Walimbe, V., \& Jagadeesh, J. M. (2003). HighSpeed Registration of Three-and Four-dimensional Medical Images by Using Voxel Similarity 1. Radiographics, 23(6), 1673-1681.

85. Zaidi, H. (2006). Quantitative analysis in nuclear medicine imaging (pp. 141-165). New York: Springer.

86. Slomka, P. J., Radau, P., Hurwitz, G. A., \& Dey, D. (2001). Automated three-dimensional quantification of myocardial perfusion and brain SPECT. Computerized medical imaging and graphics, 25(2), 153-164.

87. Hutton, B. F., Buvat, I., \& Beekman, F. J. (2011). Review and current status of SPECT scatter correction. Physics in medicine and biology, 56(14), R85.

88. Garcia, E. V., Faber, T. L., Cooke, C. D., Folks, R. D., Chen, J., \& Santana, C. (2007). The increasing role of quantification in clinical nuclear cardiology: the Emory approach. Journal of nuclear cardiology, 14(4), 420-432.

89. Cerqueira, M.D., Weissman, N.J., Dilsizian, V., Jacobs, A.K., Kaul, S., Laskey, W.K., Pennell, D.J., Rumberger, J.A., Ryan, T., Verani, M.S. (2002). Standardized myocardial segmentation and nomenclature for tomographic imaging of the heart a statement for healthcare professionals from the cardiac imaging committee of the Council on Clinical Cardiology of the American Heart Association. Circulation, 105(4), 539-542.

90. Hesse, B., Lindhardt, T.B., Acampa, W., Anagnostopoulos, C., Ballinger, J., Bax, J.J., Edenbrandt, L., Flotats, A., Germano, G., Stopar, T.G., Franken, P., Kelion, A., Kjaer, A., Le Guludec, D., Ljungberg, M., Maenhout, A.F., Marcassa, C., Marving, J., McKiddie, F., Schaefer, W.M., Stegger, L. \& Underwood, R. (2008). EANM/ESC guidelines for radionuclide imaging of cardiac function. European journal of nuclear medicine and molecular imaging, 35(4), 851-885. 
91. Slomka, P. J., \& Baum, R. P. (2009). Multimodality image registration with software: state-of-theart. European journal of nuclear medicine and molecular imaging, 36(1), 44-55.

92. Cherry, S. R. (2009, September). Multimodality imaging: Beyond pet/ct and spect/ct. In Seminars in nuclear medicine (Vol. 39, No. 5, pp. 348-353). WB Saunders.

93. Hosntalab, M., Babapour-Mofrad, F., Monshizadeh, N., \& Amoui, M. (2012). Automatic left ventricle segmentation in volumetric SPECT data set by variational level set. International journal of computer assisted radiology and surgery, 7(6), 837-843.

94. Behloul, F., Lelieveldt, B. P. F., Boudraa, A., Janier, M. F., Revel, D., \& Reiber, J. H. C. (2001). Neuro-fuzzy systems for computer-aided myocardial viability assessment. Medical Imaging, IEEE Transactions on, 20(12), 1302-1313.

95. Germano, G., Kavanagh, P.B., Waechter, P., Areeda, J., Van Kriekinge, S., Sharir, T., Lewin, H.C. \& Berman, D.S. (2000) A new algorithm for the quantitation of myocardial perfusion SPECT. I: technical principles and reproducibility. Journal of nuclear medicine?: official publication, Society of Nuclear Medicine 41, 712-9.

96. Sharir, T., Germano, G., Waechter, P.B., Kavanagh, P.B., Areeda, J.S., Gerlach J, Kang, X., Lewin, H.C. \& Berman, D.S. (2000). A new algorithm for the quantitation of myocardial perfusion SPECT. II: validation and diagnostic yield. Journal of Nuclear Medicine, 41(4), 720-727.

97. Rudd, J.H., Warburton, E.A., Fryer, T.D., Jones, H.Á., Clark, J.C., Antoun, N., Johnström, P., Davenport, A.P., Kirkpatrick, P.J., Arch, B.N., Pickard, J.D., Weissberg, P.L. (2002). Imaging atherosclerotic plaque inflammation with [18F]-fluorodeoxyglucose positron emission tomography. Circulation, 105(23), 2708-2711.

98. Takalkar, A. M., El-Haddad, G., \& Lilien, D. L. (2008). FDG-PET and PET/CT-Part II. The Indian journal of radiology \& imaging, 18(1), 17.

99. Ci'žek, J., Herholz, K., Vollmar, S., Schrader, R., Klein, J., \& Heiss, W. D. (2004). Fast and robust registration of PET and MR images of human brain. Neuroimage, 22(1), 434-442.

100. Fung, G., \& Stoeckel, J. (2007). SVM feature selection for classification of SPECT images of Alzheimer's disease using spatial information. Knowledge and Information Systems, 11(2), 243-258.

101. Buckner, R.L., Snyder, A.Z., Shannon, B.J., LaRossa, G., Sachs, R., Fotenos, A.F., Sheline, Y.I., Klunk, W.E., Mathis, C.A., Morris, J.C. \& Mintun MA (2005). Molecular, structural, and functional characterization of Alzheimer's disease: evidence for a relationship between default activity, amyloid, and memory. The Journal of Neuroscience, 25(34), 7709-7717.

102. Badiavas, K., Molyvda, E., Iakovou, I., Tsolaki, M., Psarrakos, K., \& Karatzas, N. (2011). SPECT imaging evaluation in movement disorders: far beyond visual assessment. European journal of nuclear medicine and molecular imaging, 38(4), 764-773. 